Document downloaded from:

http://hdl.handle.net/10251/166746

This paper must be cited as:

Barrachina Martínez, I.; Vivas-Consuelo, D.; Reyes-Santias, F. (2020). Cost-utility model of brivaracetam in the adjunctive treatment of patients with epilepsy in Spain. Expert review of pharmacoeconomics \& outcomes research (Online). 1-10.

https://doi.org/10.1080/14737167.2021.1838899

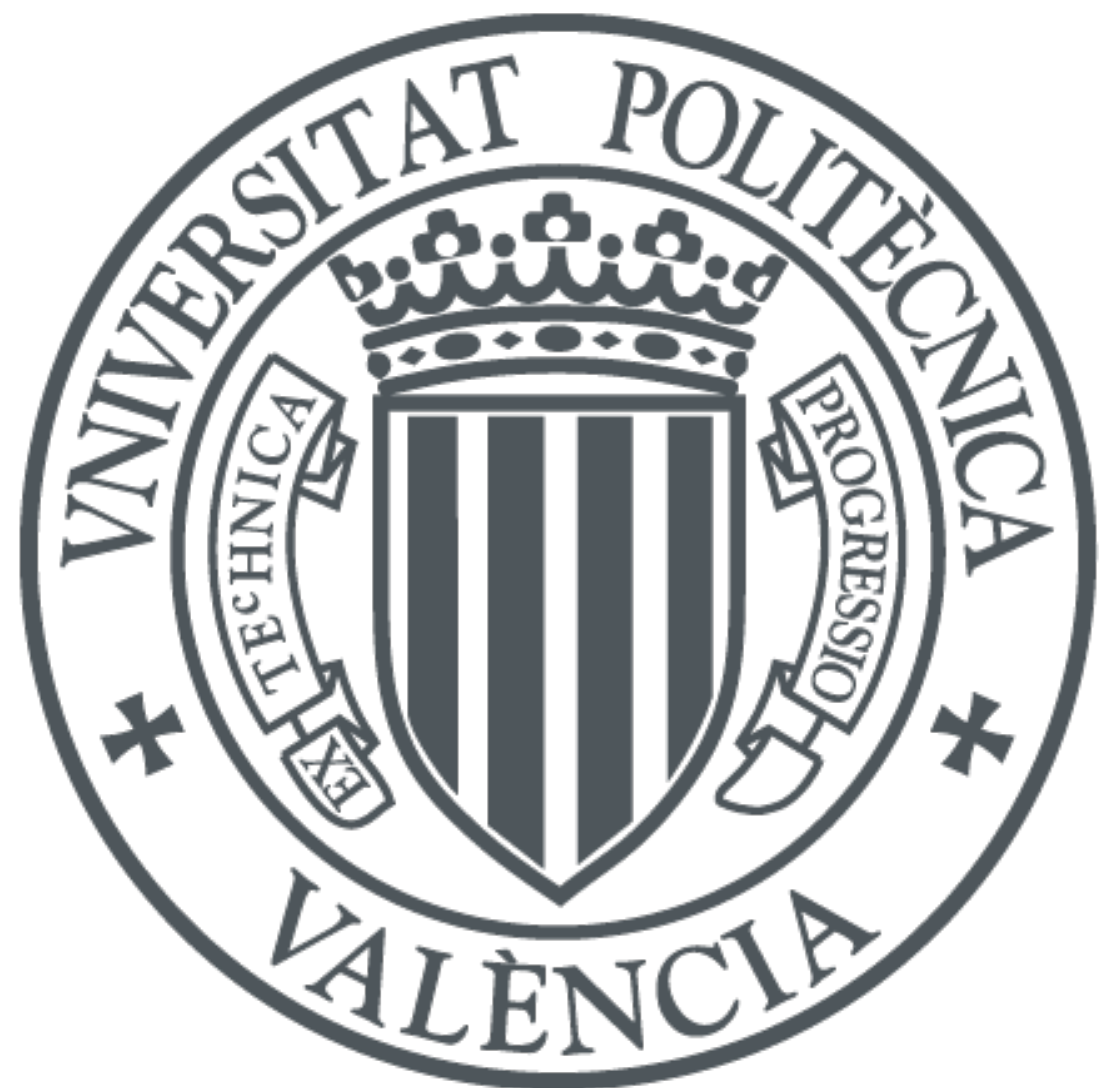

The final publication is available at

https://doi.org/10.1080/14737167.2021.1838899

Copyright Taylor \& Francis

Additional Information 


\title{
Cost-utility model of brivaracetam in the adjunctive treatment of patients with epilepsy in Spain
}

\author{
Isabel Barrachina-Martineza ${ }^{a}$ David Vivas-Consuelo and Francisco Reyes-Santias ${ }^{\mathrm{b}}$
}

\begin{abstract}
aINECO, Universitat Politècnica De Valencia, Spain; 'bepartamento De Organización De Empresas Y Marketing, Universidad De Vigo, Facultad De Ciencias Empresarias E Turismo, Ourense, Spain
\end{abstract}

\begin{abstract}
Objective: This study aims to assess the cost utility of Brivaracetam compared with the third-generation anti-epileptic drugs used as standard care.

Methods: A cost utility analysis of Brivaracetam was carried out with other third-generation comparators. The treatment pathway of a hypothetical cohort over a period of 2 years was simulated using the Markov model. Data for effectiveness and the QALYs of each health status for epilepsy, as well as for the disutilities of adverse events of treatments, were analyzed through a studies review. The cost of the anti-epileptics and the use of medical resources linked to the different health statuses were taken into consideration. A probabilistic sensitivity analysis was performed using a Monte Carlo simulation.

Results: Brivaracetam was shown to be the dominant alternative, with Incremental Cost Utility Ratio (ICUR) values from $-11,318$ for Lacosamide to $-128,482$ for Zonisamide. The probabilistic sensitivity analysis validates these results. The ICUR sensitivity is greater for increases in the price of Brivaracetam than for decreases, and for Eslicarbizapine over the other adjunctives considered in the analysis.

Conclusions: Treatment with Brivaracetam resulted in cost effective and incremental quality adjusted life years come at an acceptable cost.
\end{abstract}

ARTICLE HISTORY

Received 20 June 2020

Accepted 15 October 2020

KEYWORDS

Cost utility; anti-epileptic drugs; adjunctive therapy; markov model; monte Carlo simulation

\section{Expert opinion section}

Epilepsy is among the most common neurological disorders, with a global prevalence of almost 50 million [1] and an annual incidence of 50.4 per 100,000 persons [2], higher for low- and middle-income countries (81.7/100,000 inhabitants) than for high-income countries (45.0/100.000 inhabitants).

Patients with epilepsy suffer a multidimensional deterioration in their quality of life that has negative effects in aspects such as education, employment, and driving vehicles, as well as being affected in the functions of daily life, given that those who manage to control the disorder through medication suffer from tiredness and problems of memory and concentration among others [3] as well as having a higher probability of early death than the rest of the population [4].

As a simplistic analysis, there are two types of epileptic seizures - generalized (involving the whole surface of the brain simultaneously) and focal (beginning in one part of the brain). The second type, partial-onset seizures (POS), have more prevalence, accounting for $60 \%$ of the epileptic patients in Spain [5].

The most common treatment is therapy with oral antiepileptics (AED). These AEDs have evolved, and the newer generations are safer and better tolerated by patients. This pharmacological treatment begins with monotherapy, although this does not result in adequate control for the $30 \%$ of patients $[6,7]$ who suffer from more persistent or refractory epilepsy [8]. For patients with this condition an adjunctive therapy (add-on) is introduced as part of their maintenance therapy. The add-on choice is based on effectiveness (control of seizures, less seizures), tolerability profile, and low-risk drugs-interaction. In the case of patients who suffer from persistent seizures or chronic medicinal adverse effects, treatments that are apparently more 'aggressive', such as surgery, should be considered [9].

Nowadays there are more than 20 AEDs available for the treatment of epilepsy, from first to third-generation. Some of these have numerous side effects and interactions that can complicate patient treatment and management, especially for those with refractory epilepsy. The lack of knowledge of the causes of epileptic seizures makes the choice of medication difficult, and it is hoped that advances in genome analysis provide better understanding of AED applicability in patients [7]. Also ketogenic dietis an alternative therapeutic approach to antiepileptic drug refractoriness [10].

Brivaracetam (BRV) [11] is one of the third-generation AEDs being used as an adjunctive drug for patients between 16 and 65 years old with POS, approved in Spain in January 2016 [12]. BRV is a derivative of levetiracetam but exhibits a 20 -fold higher affinity and a faster brain entry time as a synaptic vesicle glycoprotein $2 \mathrm{~A}$ (SV2A) ligand. An additional advantage compared to other third generation AEDs is that BRV is available as an intravenous formulation providing fast penetration into the brain [13]. 
The aim of this study is to perform a cost utility analysis of BRV compared with the other AEDs used as adjunctives in the treatment of epilepsy. Specifically, comparison was with the following third generation AEDs: Eslicarbazepine, Lacosamide, Perampanel, and Zonisamide and the relation between cost increment and quality of life increment was determined for the different treatments.

\section{Methods}

\subsection{Design and analysis perspective}

A Markov model was developed using healthcare system and societal perspectives for the cost utility study of BRV with regard to the AED comparators used as adjunctives in patients over 16 diagnosed with POS. This model simulated the predicted changes in health status according to the transition probabilities of each AED within a time horizon of 8 trimesters. Transition probabilities were obtained from randomizedcontrolled trials that reported the percentage of patients who had partial ( $>50 \%$ reduction in seizure frequency) and complete responses, and the percentage of patients who discontinued treatment for any reason. Figure 1 represents the clinical pathway which is fundamental, given that the Markov models are structured around health statuses and movements between them.

Utility was expressed in terms of quality-adjusted life years (QALYs). Adverse effects of the treatments were taken into account as regards the disutilities produced. The expenditure was obtained from the costs of the different AEDs and costs derived from the use of resources implied by the different health statuses.
The main assessment metric is the incremental cost utility ratio (ICUR) of BRV acting as add-on compared with each of the other drugs. The cost effectiveness analysis was undertaken from the perspective of the National Health System.

\subsection{Treatment strategies}

The treatments compared with BRV are $3^{\text {rd }}$ generation AEDs, specifically: Eslicarbazepine, Lacosamide, Perampanel, and Zonisamide. These comparators are the most recent approved treatments. Neither Pregabalin nor Tiagabine were included as their use as an add-on treatment in Spain is infrequent.

Retigabine, one of the second-generation AEDs used as an add-on, was originally part of our comparator data [14]. As the data used is from retrospective observations and this AED was taken off the market on the 3rd of October 2016, it was decided to remove Retigabine from our data pool in order not to interfere in extrapolated results. The manufacturer of Retigabine, GlaxoSmithKline, announced a permanent stop to the manufacturing of this drug from June 2017 due to commercial reasons. In 2013, the Food and Drug Administration (FDA) had warned the public that this drug could cause blue skin discoloration and eye abnormalities characterized by pigment changes in the retina. These Adverse Events (AEs) are still being studied; currently the FDA does not know if these changes are reversible.

\subsection{Transition probabilities}

The response to treatments, or effectiveness, is measured through the transition probabilities that predict how people will move from one health status to another according to the medication administered. These are fundamental in

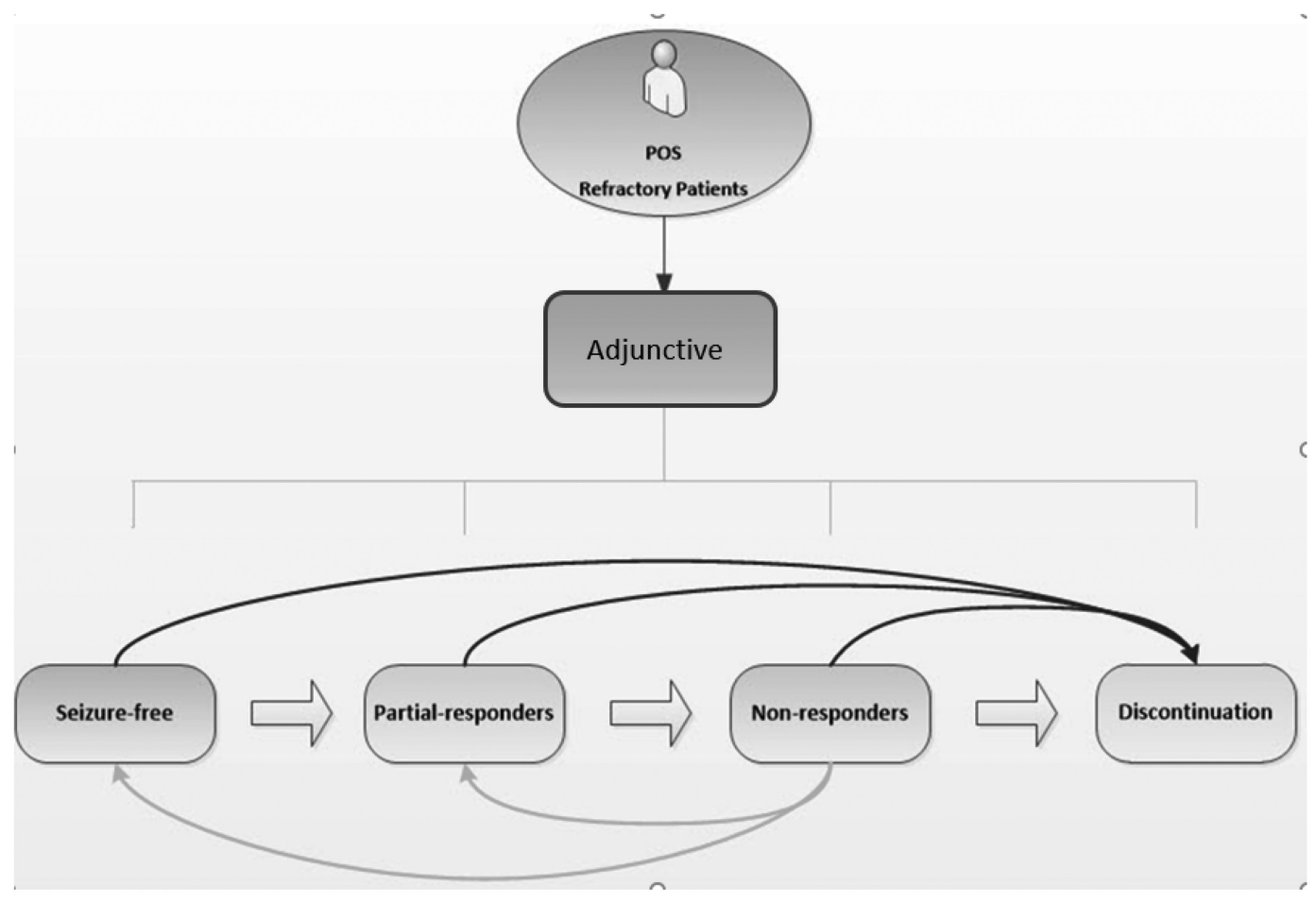

Figure 1. Markov chain model. 
determining how patients evolve in the model as the cycles progress. Moreover, the different AEDs can produce various adverse effects, with the most common being considered in this study, which are: ataxia, dizziness, fatigue, nausea, and somnolence (Table 1). These transition probabilities for each of the treatments were obtained from the Global CostEffectiveness and Disease Pathway Model for Epilepsy: Technical Report (Mishra et al. 2015), which in turn is taken from the Net Meta-analysis NMA carried out by the UCB laboratory. This report compares BRV, used as adjunctive therapy, to other specified AEDs, and can thus be used to provide evidence of comparative effectiveness. Bayesian NMAs were performed for each result using Markov Chain Monte Carlo (MCMC) methods. This approach combines previous distributions with observed data to create a back distribution as a basis for summary results [15]. As the transition probabilities of each treatment were calculated taking into account an annual scope, to convert them to 4 periods $(n=4)$ of 3-months (the usual follow-up period for chronic diseases, with neurologist visits and other examinations if needed) we used the Eigendecomposition approach [16].

\subsection{Utility data}

To measure utility, the QALYs of each health status [17] were used, together with the utility reduction for each adverse event [18]. The values were obtained from the study by Mulhern et al. (2014) carried out on 1,611 patients treated with different AEDs in monotherapy over a period of 2 years. The values of these utilities are shown in Table 2. It can be seen that the probabilities of presenting adverse effects act as disutilities, or a decrease in the quality of life of the patients, and were obtained from the work of Vera-Llonch et al [19].

\subsection{Cost estimation}

The treatment costs were calculated on the basis of the daily cost of the treatments according to the recommended dosage for each drug (Table 2). In addition, the costs of health service use were also taken into account, as well as others implied in the treatment of the disorder, such as: in-patient care, emergency visits, outpatient visits to the neurologist, visits to the general practitioner and electroencephalograms. For these costs, it is considered that the probability of resource use by the patient varies according to whether they are seizure-free, have achieved a reduction of $50 \%$ in seizures, or have either not responded to or have stopped treatment for some reason (Table 3). The trimestral costs were obtained from the frequency of health service use according to the health status of the patient, the unit cost of each service and the mean number of seizures suffered by the patient.

\subsection{Incremental cost utility ratio (ICUR)}

The outcome of the cost utility analysis is presented as an incremental cost utility ratio (ICUR). The ICUR measures the

Table 2. Drug cost (Daily cost $€$ ), utility of each patient's health status and utility reduction of each adverse events.

\begin{tabular}{lr}
\hline Drug & Cost per day $(€)$ \\
\hline Brivaracetam & 4.48 \\
Eslicarbazepine & 4.48 \\
Lacosamide & 4.48 \\
Perampanel & 4.20 \\
Zonisamide & 5.92 \\
Patients Health Status & Utility \\
Seizure-free & 0.869 \\
Partial-Response (Reduction 50\% seizures) & 0.805 \\
Non - responder & 0.683 \\
Adverse Events & -0.280 \\
Ataxia (coordination loss) & -0.220 \\
Dizziness & -0.250 \\
Fatigue & -0.230 \\
Nausea & -0.220 \\
Somnolence & 0.60 \\
\hline
\end{tabular}

Average cost of concomitant monotherapy (CZP, LMT, OXC, TPR, VAL): 0, $60 €$ per day.

incremental cost divided by the incremental utility of each AED compared to BRV. The ICUR calculation is given by the formula:

$$
I C U R=\frac{C_{2}-C_{1}}{U_{2}-U_{1}}=\frac{\Delta C}{\Delta U}
$$

Where:

$\mathrm{C} 2$ is the cost under BRV

Table 1. Transition probabilities of each adjunctive treatment (AT) and adverse events annual probabilities.

\begin{tabular}{|c|c|c|c|c|c|}
\hline \multicolumn{6}{|l|}{ Effectiveness of each AT } \\
\hline & Brivaracetam & Eslicarbazepine & Lacosamide & Perampanel & Zonisamide \\
\hline $\begin{array}{l}\text { Responders } \\
\quad \text { (Seizure-free) }\end{array}$ & 0.065 & 0.032 & 0.03 & 0.035 & 0.018 \\
\hline $\begin{array}{r}\text { Partial Responders } \\
\text { (50\% Response) }\end{array}$ & 0.355 & 0.33 & 0.308 & 0.296 & 0.335 \\
\hline $\begin{array}{l}\text { Non-responders } \\
(1 \text {-(others probability)) }\end{array}$ & 0.370 & 0.469 & 0.448 & 0.493 & 0.47 \\
\hline $\begin{array}{l}\text { Discontinuation } \\
\text { (any reason) }\end{array}$ & 0.210 & 0.169 & 0.214 & 0.176 & 0.177 \\
\hline \multicolumn{6}{|l|}{ Adverse Events } \\
\hline & Brivaracetam & Eslicarbazepine & Lacosamide & Perampanel & Zonisamide \\
\hline Ataxia & 0.03 & 0.06 & 0.08 & 0.04 & 0.031 \\
\hline Dizziness & 0.13 & 0.23 & 0.24 & 0.23 & 0.088 \\
\hline Fatigue & 0.11 & 0.06 & 0.06 & 0.07 & 0.054 \\
\hline Nausea & 0.05 & 0.12 & 0.11 & 0.01 & 0.053 \\
\hline Somnolence & 0.14 & 0.12 & 0.1 & 0.14 & 0.078 \\
\hline
\end{tabular}


Table 3. Healthcare costs.

\begin{tabular}{|c|c|c|c|c|}
\hline \multicolumn{5}{|l|}{ Probability of service use } \\
\hline & Seizure free & $\begin{array}{l}\text { Partial response } \\
\text { (50\% reduction) }\end{array}$ & Non- responder & Discontinuation \\
\hline Service & 6 months use & 6 months use & 6 months use & 6 months use \\
\hline Inpatient care (\%) & $0.30 \%$ & $0.30 \%$ & $0.30 \%$ & $0.30 \%$ \\
\hline Emergency visit (\%) & $0.80 \%$ & $0.80 \%$ & $0.80 \%$ & $0.80 \%$ \\
\hline Outpatient visit to neurologist & 0.75 & 0.82 & 1.02 & 4.52 \\
\hline GP visit & 0.08 & 0.14 & 0.34 & 0.14 \\
\hline EEG & 0.3 & 0.3 & 0 & 0 \\
\hline \multicolumn{5}{|l|}{ Unit cost service } \\
\hline Service & Unit cost & & & \\
\hline Inpatient care & $2,029.90$ & & & \\
\hline Emergency visit & 202.39 & & & \\
\hline Outpatient visit to neurologist & 92.62 & & & \\
\hline GP visit & 89.93 & & & \\
\hline EEG & 55.26 & & & \\
\hline \multicolumn{5}{|l|}{ Service cost } \\
\hline & \multicolumn{4}{|c|}{ Number of crisis } \\
\hline & Seizure free & $\begin{array}{l}\text { Partial response } \\
\text { (50\% reduction) }\end{array}$ & Non- responder & Discontinuation \\
\hline Service & 0 & 40 & 160 & 160 \\
\hline Inpatient care & 0 & 244 & 974 & 974 \\
\hline Emergency visit & 0 & 65 & 259 & 259 \\
\hline Outpatient visit to neurologist & 69 & 76 & 94 & 419 \\
\hline GP visit & 7 & 13 & 31 & 13 \\
\hline EEG & 16,578 & 16,578 & 0 & 0 \\
\hline 6 months cost & 93 & 413 & 1.358 & 1.665 \\
\hline 3 months cost & 47 & 207 & 679 & 832 \\
\hline
\end{tabular}

U2 is the effectiveness under BRV

$\mathrm{C} 1$ is the cost under the comparator

$\mathrm{U} 1$ is the effectiveness under comparator

The cost utility plane shows the results obtained for each AED compared to BRV. The y-axis shows the cost difference while the $x$-axis shows the utility difference. The position on this plane gives the dominance of the anti-epileptic.

These ICUR analyses were performed through the Markov chain model, a model well suited for use in epilepsy, as it can integrate the time factor. The Markov model represents the natural evolution of epilepsy considering the health status changes (Figure 1) and adverse events relating these with cost-utility for each treatment with a two year time horizon and trimestral cycle length (a total of 8 trimesters), where the patients are transiting from one status to another or are maintained stable.

The cost effectiveness or cost utility analysis requires a modeling to be carried out as a systematic focus for decision making in uncertain conditions where the probability of every possible event, together with their consequences, is explicitly established. Decision trees are the most useful type of decision modeling when health events happen close together and do not repeat, when health events happen quickly or not at all, and when uncertainty over the effects of treatment is resolved quickly. Markov models are more complex than decisions trees. They enable us to address a wide range of questions, and furthermore can explicitly take account of time. This is especially useful in chronic diseases, as the clinical evolution of patients and their health status, can pass through different stages over time.

The simulation Markov model was made using Microsoft Excel and was provided with: 1) treatment effectiveness (transition probabilities of responding to each treatment); 2) treatment cost; 3) utility (QALYs weight).
The data obtained is based on a specified cohort of 1 patient. These calculations, as before, were performed for 8 cycles, each of three months.

As the time horizon for the study was over one year, the economic evaluation was conducted with a discount rate of 3 $\%$. This was applied to both costs and health effects as recommended [20].

\subsection{Sensitivity analyses}

The one way sensitivity analysis only determines the influence of price variation of BRV on the results of the analysis. The probabilistic sensitivity analysis (PSA), however, evaluates the effect of the simultaneous variation of the different parameters introduced into the model in the results of the cost utility analysis of BRV compared to the other AEDs.

This uses the random change of variables, in which the model is based on studying the impact of each of these on the results.

To calculate the PSA the $\beta$ statistical distribution (values 0-1 range) is used for random calculation of the effectiveness of treatments, and the lognormal statistical distribution for random calculation of the cost.

The $\beta$ statistical distribution depends on the parameters ( $a$, $\beta)$. QALY weights assume that $a=\mu^{*} n$ where $\mu$ is the mean and $\mathrm{n}$ is the number of observations and $\beta=(1-\mu) n$. However, health costs supposedly follow a lognormal distribution instead of a $\beta$ distribution as QALY weights.

The sensitivity analysis was carried out through a Monte Carlo simulation, a computerized mathematical technique that randomly determines the possible results of the analysis from the variables used as input (cost) and output (utility), 
randomly extracted from the possible values allowed according to the distribution function from which they come.

The impact of the health status switching threshold was tested separately, with each AED medication determining the treatment duration for each intervention in the model. The BRV as add-on treatment effectiveness on clinical parameters was applied for the treatment period of 1 and 2 years. The effect of having fewer seizures was expected to be an important driver of the cost effectiveness and cost utility results, given the impact of the adjunctive drug on patients' AED treatment maintenance, with less seizures, AEs, and healthcare costs.

\section{Results}

\subsection{Base-case analysis}

Table 4 shows the results for effectiveness, utility, and cost for each AED, as well as the incremental values for BRV compared to the alternative adjunctive treatments applied to a single patient and the ICUR and ICER ratios.

The ICUR goes from $-128,482$ to $-11,318 €$ per QALY gained and the ICER goes from $-12,827$ to $-3,817 €$ per QALY gained.

Figure 2 simultaneously shows the points of increase in effectiveness and in utility compared with the increase in cost for patients treated with BRV compared to the other AEDs referred to in the study. It can be seen that both series of points are represented in the lower right quadrant, which means that it gives better results both regarding effectiveness and utility and a lower cost.
The reduction in differences in results is greater when considering effectiveness than utility, due to the units of measurement. In the case of Zonisamide, the greatest difference in results is seen according to whether effectiveness or utility is being evaluated, as the improvement in utility is much lower than the improvement in effectiveness, which may be due to the disutilities connected to the adverse effects, although it is still positive and the cost savings are evident. As can be seen in Table 2, BRV has a greater probability of adverse effects than Zonisamide, with the latter slightly higher only in the case of ataxia and nausea.

Figure 2 shows that Eslicarbizapine is the treatment closest in cost utility and cost effectiveness to BRV.

\subsection{Sensitivity analysis}

\subsubsection{One-way sensitivity analysis}

The one-way sensitivity analysis for $5 \%$ variations in the price of BRV shows it is still the dominant option (Table 5). Nevertheless, the effect is different according to each medicine and the percentage increases are greater for a lowering of the price of BRV than for an increase of the same amount (5\%). Figure 3 shows the percentage variation of the ICUR corresponding to a $1 \%$ variation in price of BRV upwards or downwards. Thus a modification upwards of the unit percentage point of the BRV price entails a reduction of the ICUR by $9.4 \%$ for Eslicarbacepine, 7.9\% for Lacosamide, 6.2\% for Perampanel and $1.9 \%$ for Zonisamide, whereas a modification downwards of the unit percentage point of the price entails an increase of the ICUR by $6.3 \%$ for Eslicarbacepine, $5.5 \%$ for Lacosamide, 5.0\% for Perampanel and 1.8\% for Zonisamide. (Figure 3)

Table 4. Base-case results by one patient and two years treatment.

\begin{tabular}{|c|c|c|c|c|c|c|c|c|}
\hline AED & Effect. & QALY & Cost & Effect. & Utility & Cost & ICUR & ICER \\
\hline Brivaracetam & 0.118 & 1,408 & $7,058.8$ & & & & & \\
\hline Eslicarbazepine & 0.060 & 1,390 & $7,279.7$ & 0.058 & 0.018 & -220.91 & $-12,500$ & $-3,817$ \\
\hline Lacosamide & 0.055 & 1,384 & $7,323.7$ & 0.062 & 0.023 & -264.93 & $-11,318$ & $-4,242$ \\
\hline Perampanel & 0.066 & 1,390 & $7,384.0$ & 0.052 & 0.018 & -325.23 & $-18,085$ & $-6,250$ \\
\hline Zonisamide & 0.034 & 1,399 & $8,137.1$ & 0.084 & 0.008 & $-1,078.35$ & $-128,482$ & $-12,827$ \\
\hline
\end{tabular}

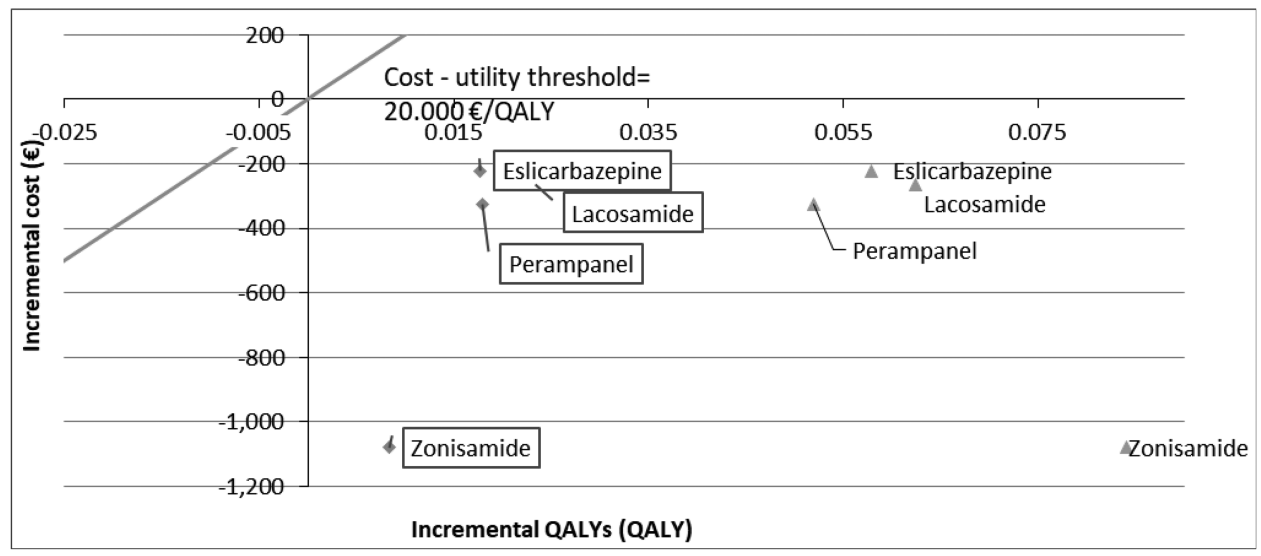

ICER + vs ICUR

Figure 2. Incremental cost effectiveness ratio ICER and incremental cost utility ratio ICUR. 
Table 5. OWA incremental cost effectiveness ratio given a $5 \%$ variation in cost of BRV.

\begin{tabular}{llll}
\hline ICUR & & \\
\hline Cost BRV $+5 \%$ & & Cost BRV $-5 \%$ & \\
Eslicarbazepine & $-6,646$ & Eslicarbazepine & $-18,354$ \\
Lacosamide & $-6,899$ & Lacosamide & $-15,739$ \\
Perampanel & $-12,332$ & Perampanel & $-23,838$ \\
Zonisamide & $-116,150$ & Zonisamide & $-140,801$ \\
\hline
\end{tabular}

\subsubsection{Multivariate sensitivity analysis}

The Monte Carlo simulation for the increase of QALYs compared to the increase in cost shows the results of the multivariate sensitivity analysis (Figure 4Figure 3 ), where the points are largely in the lower right quadrant and always below the acceptability threshold. This means that BRV achieves greater utility and lower costs than the alternatives, implying it would be the dominant option (Figure 5).

An additional path to the cost utility plane when presenting the results of a probabilistic sensitivity analysis is to draw up the cost utility acceptability curve, which is a graphical representation of the quantification of the uncertainty around the expected cost utility. This shows the probability of acceptability according to the established threshold or willingness to pay.

This resulted in an ICER point estimate of 20,000 Euros per QALY gained. The sensitivity analysis determined incremental costs to be sensitive to BRV price and the treatment effect on treatment-response having a notable impact on the incremental QALYs. However, there were no scenarios that raised the ICER above 20,000 Euros per QALY.

\section{Discussion}

This study shows the usefulness of Markov chains in the costefficiency analysis of adjunctive treatment for epilepsy, although this is extendable to other treatments. The available data on treatment costs and use of resources derived from the disorder are compared in the cost effectiveness analysis and utility in the cost utility analysis. The results indicate that BRV is a dominant option with regards to the comparators and the sensitivity analysis places all the points in the lower right quadrant, above all in the cost effectiveness analysis. For the cost utility, the differences are less notable, due to the greater presence of adverse effects presented by BRV over the

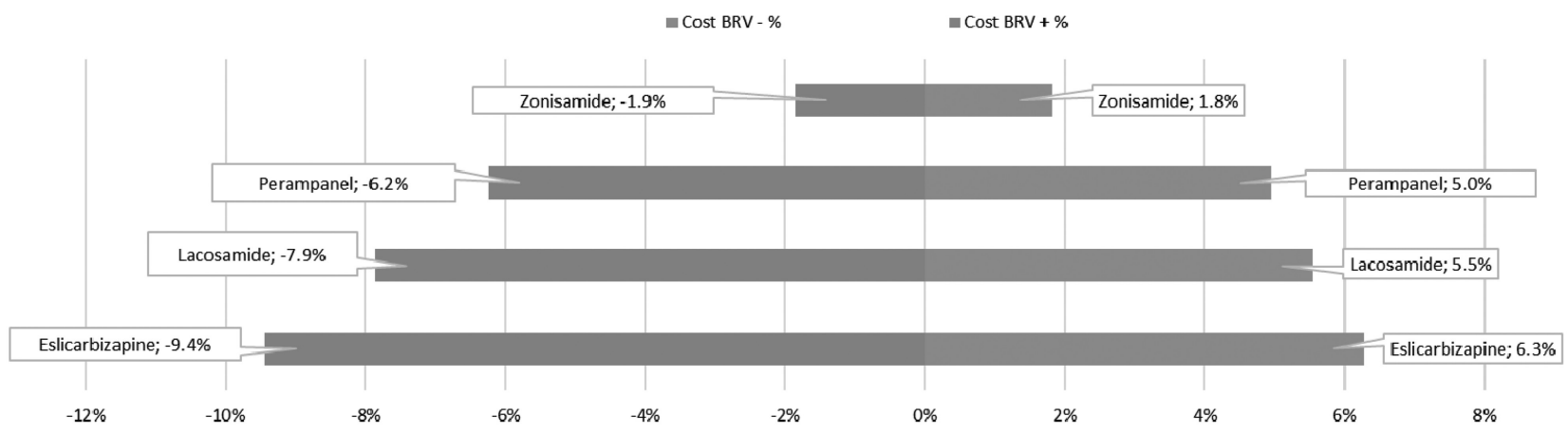

Figure 3. Percentage differences in the ICUR with $1 \%$ variation in cost of BRV.

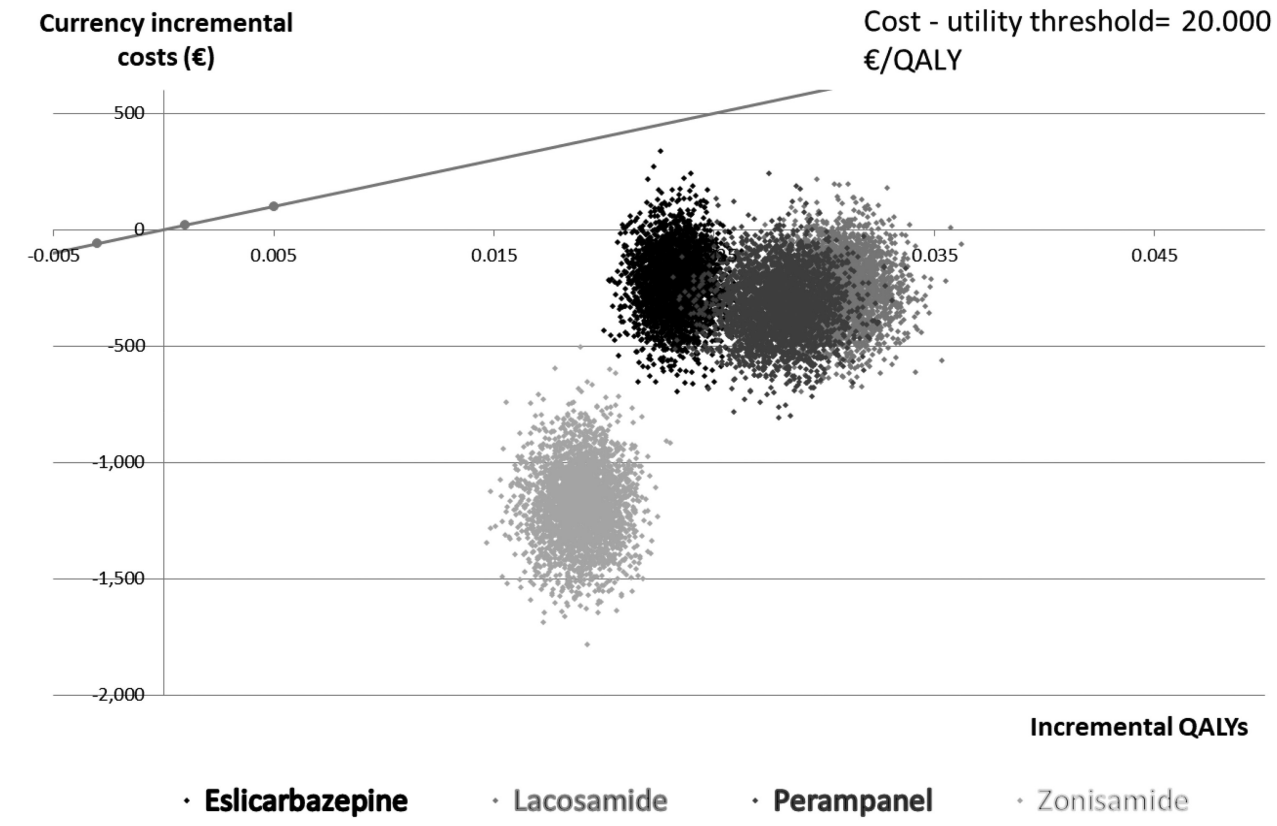




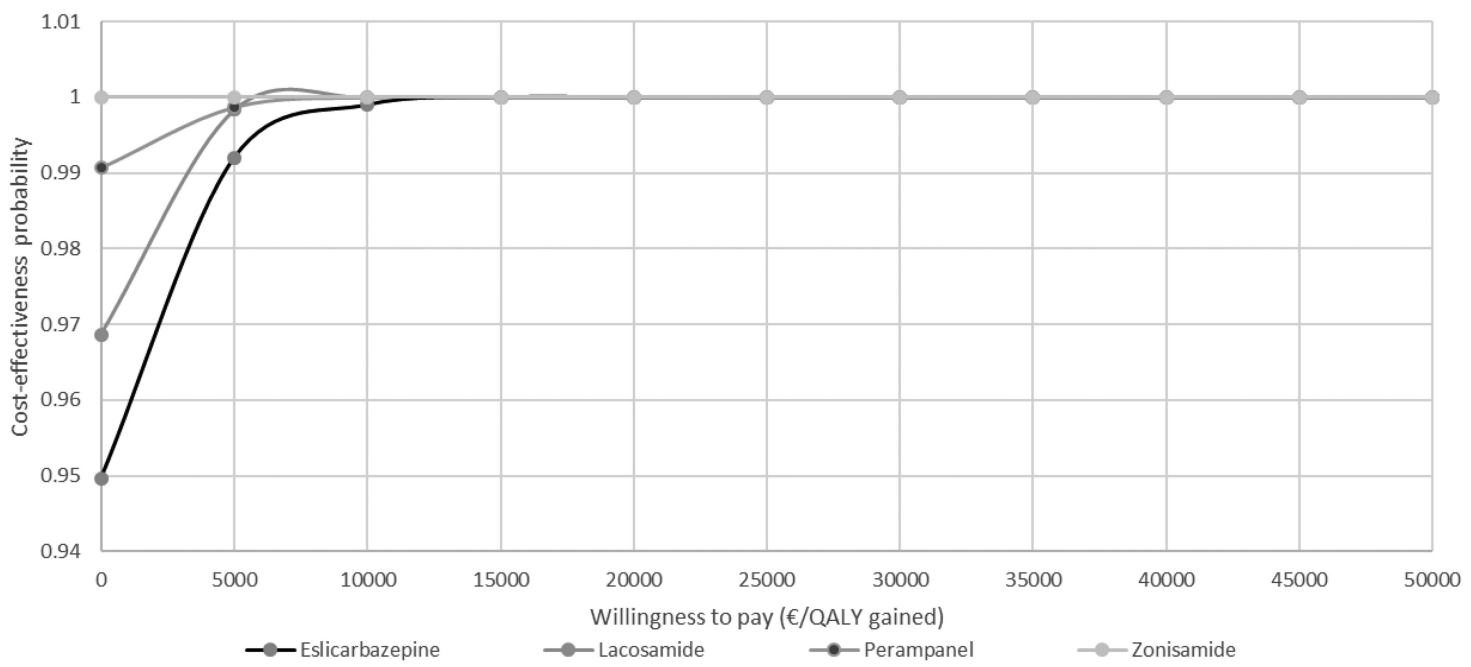

Figure 5. Acceptability curve for one patient comparing BRV versus each alternative treatment.

comparators. Regarding this point, it is worth considering that not all the adverse effects detected as deriving from these drugs were taken into account, but only the most frequent, which gives rise to the possibility that the results would have been different if all those detected had been introduced.

The budgetary impact study for the introduction of BRV into the portfolio of anti-epileptic drugs for patients over 16 with POS (Barrachina et al) (2018) shows savings of $0.23 \%$ of the cost of adjunctive treatments for epilepsy over five years. A probabilistic sensitivity analysis performed through a Monte Carlo simulation where all the variables introduced into the budget impact analysis varied simultaneously estimated that the probability of savings for the National Health Service was $84 \%$ [21].

The increasing number of treatment options and the high costs associated with epilepsy underline the need for economic evaluations in this field [22]. With regard to existing cost effectiveness and cost utility studies for epilepsy treatments, it is very difficult to compare results due to the different models used in the economic evaluations and the different parameters employed in the studies [23].The results in the present study were represented analyzing studies using the recommended methodologies that discuss the effectiveness and costs.

Cost evaluation is very important in benefiting patients, allowing healthcare costs payers to be well aware of their options. The treatment for patients with drug-resistant epilepsy should manage to render them seizure-free, avoid treatment-related adverse effects, and allow the individual to become a participating and productive member of society. In this paper effectiveness and cost data were obtained from the literature, but also included other healthcare costs derived from each health status, while most cost effectiveness analyses restrict the scope of costs to drugs

The available AEDs and utility values directly related to the treatment used were modeled, but the indirect costs and other clinically important epilepsy attributes (e.g.,seizure severity, side-effects of crisis) and patient adherence in terms of each treatment convenience in refractory patients, are missing [23].

Regarding the methodology used in the present study, it could be said that other authors [24] have concluded that decision trees and Markov models both behave in a similar way and that the differences between them are very small. However, they considered that a decision tree is more suitable for generalized samples learned in a specific context, while a Markov model is more suitable when the exact learning sample must be recalled. Moreover, a decision tree may sometimes generate false suggestions.

As in the current study, Kotirum et al (2017) [25]developed a decision tree combined with a Markov model to simulate costs and health outcomes covering a lifetime horizon in societal and healthcare payer perspectives. However, unlike the present study, a multivariate probabilistic sensitivity analysis (PSA) was also conducted by these authors to simultaneously examine the effects of all parameter uncertainty using a Monte Carlo simulation, although some studies have shown that Markov models are not sensitive to the uncertainty in the associated transition probability matrices [26].

On the other hand, Swalow et al (2017) [27] conducted a study on the propensity for enrollment in the BRV versus levetiracetam trials, using a different approach than our method, and estimates a multivariate logistic regression model where baseline characteristics were independent variables. Several propensity score models were evaluated for model fit. The referred study showed that placebo arm differences in adjunctive BRV and levetiracetam epilepsy drug trials indicate the presence of unobserved confounding factors associated with placebo response. However, other studies show that the estimated Markov switching models result in a superior statistical fit relative to the standard (single-state) multinomial logit models [28] and that the choice probabilities computed by the Markov chain-based model are a good approximation to the true choice probabilities for any random utility-based choice model under mild conditions.

Hawkins et al (2005) [29] evaluated the decision model developed for the National Institute for Clinical Excellence 
(NICE) in the United Kingdom, comparing the long-term cost effectiveness of drugs licensed in adults. Benefits were assessed in terms of QALYs. Costs and benefits were discounted at $6 \%$ and $1.5 \%$, respectively, per annum, the NICE proposal. In the present study we discount at $3 \%$ as the Washington Panel proposes and also discount costs, reduced number of seizures and QALYS at the same rate [30].

Unlike our study, Hawkins et al took into account for the analysis of the full range of pharmaceutical therapies feasibly used in the UK health system. In the case of our present study, only the most used drugs in Spain have been selected for comparison with BRV [31], as do other authors [32].

The results of the present study, which shows a higher cost effectiveness ratio than utility cost, is not uncommon in economic evaluation studies $[19,[33,34]$

In addition, this may be due to the ability of the QALYs to capture the impact of adverse effects. Considering the adverse effects causes the utility of the treatments to vary, due to which the results obtained when comparing cost effectiveness differ from those of cost utility. Therefore, it must be taken into account that the adverse effects of the treatments considered in this study are those that are the most common in the drugs selected for comparison [35-37]. In that sense, authors like Sherina et al (2017) [38] obtain results in which epilepsy is associated with not only seizures but also a variety of serious co-morbidities and therefore, as other complications increase, the expenditure will also increase, although the cost due to adverse events is higher with older AED's than newer drugs.

In this regard and in relation to our results in cost effectiveness and cost utility, the same authors obtain results indicating that older drugs are equally efficacious as compared to the newer in controlling seizure frequency and improving the quality of life, but are more cost effective.

Finally, it should be noted that authors such as Charokopou et al (2019) [15] warn that although the results of their network meta-analysis based on 65 published RCTs of BRV and other AEDs in patients with focal seizures would appear to show the relative equivalence in efficacy, safety, and tolerability outcomes of the included AEDs, it must be kept in mind that, due to the inability of network meta-analysis in epilepsy to capture unmeasured confounding factors and population heterogeneity, network meta-analysis should not be the sole basis for comparative treatment recommendations. Healthcare policymakers might also consider real-world experience to mitigate study generalizability concerns, and an individualized treatment approach to alleviate the approach of pooling populations together in clinical studies, to which the present study tries to contribute.

\subsection{Limitations}

The limitations of these studies are the lack of real-life comparative data, with most of the data estimated [22]. Although these estimations are modeled, they can over- and underestimate probabilistic values using the healthcare payer perspective. More real-life data can give us additional information of costs. The indirect costs (productivity, concentration, patient-time loss, and the subjective resources of each patient) taking into account the physician and patient perspective, are not always used to analyze cost effectiveness/utility. Neither are the treatment costs for adverse effects considered. Much uncertainty is associated with decisions by patients and physicians to change AEDs therapies. Physicians should perform Quality of Life (QoL) studies from the patient's real-life data. These could be a key factor in physician-patient relationship, and a tool to help the physician analyze the real overall effect of the patient-therapy, not only over disease effects but others in his/her life, giving us a patient-physician perspective.

Most of the limitations of studies of chronic diseases, with probabilities of crisis with no explanation, come from the uncertainty of results depending on each patient. It is difficult to create a function/algorithm that represents all the patients' pool, especially if the data come from pivotal trials that are done in clinical trial-controlled conditions [27]. This is one of the limitations and reasons why more studies with real data (e.g. post-marketing) are important to help decision-making positions (physicians, healthcare policies, etc.) support their choice [39].

Further research and time is needed to be able to make real world data available, extrapolated to values that can model real-life observations. The economic evaluation of all perspectives (the healthcare payer, pharmaceutical, physicians and patients) would be the best approach. The major limitations on cost effectiveness/utility studies are the data, perspectives, and methodologies available. Each value that is modeled through a cascade of methodologies incorporates an error from each of these. More studies with new data and methodologies are being analyzed [40].

\section{Conclusions}

The Markov chains model is useful for the cost effectiveness and cost utility analysis of BRV compared with the following AEDs: Eslicarbacepine, Lacosamide, Perampanel and Zonisamide. This analysis has shown that from the point of view of both the ICER and the ICUR, BRV is the 'dominant option', indicating that BRV has more utility and less cost compared to the other treatments. The difference was more marked with Zonisamide than the other three comparators. This conclusion was corroborated with the PSA, which supported the strength of the study, as the results are similar to the cost utility analysis. The model could include other possible AEDs as comparators and assess switch sequences between the AEDs.

The acceptability curve is a useful tool for making decisions and assigning available resources to financing the cost effective alternatives, although it does have the limitation of not being able to distinguish different combined distributions of costs and incremental results of the options being compared.

Ultimately, the results show that the incremental QALYs come at an acceptable incremental cost when employing the commonly accepted payer willingness-to-pay threshold of 20,000 Euros per QALY gained (acceptability curve).

\section{Acknowledgments}

We would also like to thank John Wright for the English editing. 


\section{Authors" contributions}

Isabel Barrachina conceived of the study, its design, performed part of the literature review and coordinate the draft the manuscript; David Vivas participated in the design of the study, performed part of the literature review and helped to draft the manuscript; Francisco Reyes-Santias performed part of the literature review and helped to draft the manuscript.

\section{Availability of data and supporting materials section}

No additional data are available.

\section{Consent for publication}

Not applicable.

\section{Ethics approval and consent to participate}

Not applicable.

\section{Reviewers disclosure}

Peer reviewers on this manuscript have no relevant financial relationships or otherwise to disclose.

\section{Funding}

This paper was not funded.

\section{Declaration of interest}

The authors have no relevant affiliations or financial involvement with any organization or entity with a financial interest in or financial conflict with the subject matter or materials discussed in the manuscript. This includes employment, consultancies, honoraria, stock ownership or options, expert testimony, grants or patents received or pending, or royalties.

\section{References}

1. WHO | Epilepsy: aISBN public health imperative. ISBN 978-92-4151593-1. World Health Organization 2019. Printed in Thailand.
10. Giordano C, Marchiã M, Timofeeva E, et al. Neuroactive Peptides as Putative Mediators of Antiepileptic Ketogenic Diets. Front Neurol. 2014;5 (63): 1-14.

11. European Medicines Agency. Committe for Medicinal Products for Human Use (CHMP) Briviact brivaracetam. Assessment Report EMA/ CHMP/822086/2015; Nov.

12. Markham A. Brivaracetam: first global approval. Drugs. 2016;76:517-522.

13. Willems LM, Bauer S, Rosenow F, et al. Recent advances in the pharmacotherapy of epilepsy: brivaracetam and perampanel as broad-spectrum antiseizure drugs for the treatment of epilepsies and status epilepticus. Expert Opin Pharmacother [Internet]. 2019;20:1-11

14. Craig D, Rice S, Paton F, et al. Retigabine for the adjunctive treatment of adults with partial-onset seizures in epilepsy with and without secondary generalization: A NICE single technology appraisal. Pharmacoeconomics. 2013 Feb; 31(2):101-110.

15. Charokopou M, Harvey R, Srivastava K, et al. Relative performance of brivaracetam as adjunctive treatment of focal seizures in adults: a network meta-analysis. Curr Med Res Opin [Internet]. 2019;35:1345-1354

16. Chhatwal J. Changing cycle lengths in state-transition models: doing it the right way; [cited 2018 Jan 23]. Available from: https://www.ispor.org/News/Connections_methodology_statetransition-models.PDF

17. Mulhern B, Rowen D, Snape D, et al. Valuations of epilepsy-specific health states: a comparison of patients with epilepsy and the general population. Epilepsy Behav [Internet]. 2014 [cited 2018 Jun 8];36:12-17.

18. Kristian B, Wachtmeister K, Stefan F, et al. Retigabine as add-on treatment of refractory epilepsy - a cost-utility study in a Swedish setting. Acta Neurol Scand [Internet]. 2013 [cited 2018 Jan 23];127:419-426.

19. Vera-Llonch M, Brandenburg NA, Oster G. Cost-effectiveness of add-on therapy with pregabalin in patients with refractory partial epilepsy. Epilepsia. 2008;49:431-437.

20. Drummond M., Schulpher MJ, Torrance GW, et al Methods for the economic evaluation of health care programmes.Oxford University Press, 2005.

21. Barrachina-Martínez I, Vivas-Consuelo D, Piera-Balbastre A. Budget impact analysis of brivaracetam adjunctive therapy for partial-onset epileptic seizures in valencia community, Spain. Clin Drug Investig. 2018 Apr; 38(4):353-363.

22. Simoens S. Pharmacoeconomics of anti-epileptic drugs as adjunctive therapy for refractory epilepsy. Expert Rev Pharmacoeconomics Outcomes Res [Internet]. 2010;10:309-315. Available from: http://www.scopus.com/inward/record.url?eid= 2 - s $2.0-77953700981 \& p$ artne r I D = 40 \& m d $5=$ 902059295990aa17829c1460973dc23c

23. Wijnen BFM, van Mastrigt GAPG, Evers SMAA, et al. A systematic review of economic evaluations of treatments for patients with epilepsy. Epilepsia. 2017;58:706-726.

24. De Boeck J, Verpoorten K, Luyten K, et al. Comparison between decision trees and markov models to support proactive interfaces. 18th International Workshop on Database and Expert Systems Applications (DEXA 2007); Regensburg, 2007: 94-98 [cited 2019 Sep 25]. Available from: https://core.ac.uk/download/pdf/ 34524516.pdf

25. Kotirum S, Muangchana C, Techathawat S, et al. Economic evaluation and budget impact analysis of vaccination against haemophilus influenzae Type b infection in Thailand. Front Public Health [Internet]. 2017;5. [cited 2019 Sep 25]. Available from: http://jour nal.frontiersin.org/article/10.3389/fpubh.2017.00289/full

26. Zhang Y, Wu H, Denton BT, et al. Probabilistic sensitivity analysis on Markov models with uncertain transition probabilities: an application in evaluating treatment decisions for type 2 diabetes. Health Care Manag Sci [Internet]. 2019 [cited 2019 Sep 25];22:34-52.

27. Swallow E, Fang A, Signorovitch J, et al. Can matching-adjusted indirect comparison methods mitigate placebo response
6. Kwan P, Brodie MJ. Early identification of refractory epilepsy. N Engl Med. 2000;342:314-319.

7. Brodie MJ. Epilepsy: randomised trials and genetic tribulations. Lancet Neurol [Internet]. 2008;7:7-8. cited 2019 Sep 6].

8. French JA. Refractory epilepsy: one size does not fit all. Epilepsy Curr [Internet]. 2006;6:177-180. [cited 2019 Sep 6]. Available from: http://journals.sagepub.com/doi/10.1111/j.1535-7511.2006.00137.x

9. Laxer KD, Trinka E, Hirsch LJ, et al. The consequences of refractory epilepsy and its treatment. Epilepsy Behav [Internet]. 2014 [cited 2019 Sep 6];37:59-70. 
differences among patient populations in adjunctive trials of brivaracetam and levetiracetam? CNS Drugs [Internet]. 2017;31:899-910.

28. Malyshkina NV, Mannering FL. Markov switching multinomial logit model: an application to accident injury severities; 2008 [cited 2019 Sep 25]. Available from: http://arxiv.org/abs/0811.3644

29. Hawkins N, Epstein D, Drummond $M$, et al. Assessing the cost-effectiveness of new pharmaceuticals in epilepsy in adults: the results of a probabilistic decision model. Med Decis Mak [Internet]. 2005 [cited 2019 Sep 25];25:493-510.

30. Pinto Prades JL, Sánchez Martínez Fl, Abellán Perpinán JM. Métodos para la evaluación económica de nuevas prestaciones [Internet]. Ministerio de Sanidad y Consumo. 2003. [cited 2019 Sep 25]. Available from: https://dialnet.unirioja.es/servlet/libro? codigo $=114939$

31. Agencia Española del Medicamento. Utilización de medicamentos antiepilépticos en España durante el periodo 2008-2016 [Internet]; 2017 [cited 2019 Sep 25]. Available from: https://www.aemps.gob. es/medicamentosUsoHumano/observatorio/docs/antiepilepticosperiodo-2008-2016.pdf

32. Megiddo I, Colson A, Chisholm D, et al. Health and economic benefits of public financing of epilepsy treatment in India: an agent-based simulation model. Epilepsia [Internet]. 2016;57:464-474. [cited 2019 Sep 25]. Available from: http://www. ncbi.nlm.nih.gov/pubmed/26765291

33. de Andrés-nogales F, Oyagüez I, Álvarez-Sala LA, et al. Análisis coste-efectividad y coste-utilidad de apixaban frente a dabigatrán y rivaroxaban en el tratamiento y prevención secundaria del tromboembolismo venoso. PharmacoEconomics Spanish Res Artic
[Internet]. 2017;14:7-18. [cited 2019 Sep 25]. Available from: http://link.springer.com/10.1007/s40277-016-0064-8

34. Romero M, Marrugo R, Paez M. Análisis De Costo Efectividad Y Costo Utilidad De La Adición De Rivaroxabán En El Tratamiento De Pacientes Con Síndrome Coronario Agudo En Colombia. Value Heal [Internet]. 2015;18:A830. [cited 2019 Sep 25]. Available from: https://linkinghub.elsevier.com/retrieve/pii/S1098301515023906

35. Fricke-Galindo I, Jung-Cook H, LLerena A, et al. Farmacogenética de reacciones adversas a fármacos antiepilépticos. Neurología [Internet]. 2018 [cited 2019 Sep 25];33:165-176.

36. Steinhoff BJ, Bacher M, Bucurenciu I, et al. Real-life experience with brivaracetam in 101 patients with difficult-to-treat epilepsy-A monocenter survey. Seizure [Internet]. 2017 [cited 2019 Sep 25];48:11-14.

37. Chiang H-I, Lim S-N, Hsieh H-Y, et al. Preliminary Asian experience of using perampanel in clinical practice. Biomed J [Internet]. 2018 [cited 2019 Sep 25];33:347.

38. Sherina PK. Abdurahiman. current concepts and future perspectives in cost- effectiveness analysis of antiepileptic drugs : a review, Int J Pharm Pharm reseacrh, 2017 vol. 10 (3): 371-379

39. de Kinderen RJA, Wijnen BFM, van Breukelen G, et al. From clinically relevant outcome measures to quality of life in epilepsy: A time trade-off study. Epilepsy Res [Internet]. 2016;125:24-31.

40. Cortés J-C, Navarro-Quiles A, Romero J-V, et al. Randomizing the parameters of a Markov chain to model the stroke disease: A technical generalization of established computational methodologies towards improving real applications. J Comput Appl Math [Internet]. 2017 [cited 2018 Jan 10];324:225-240. 